\title{
A Facile Route of Synthesis for Making Flibanserin
}

Feipu Yang ${ }^{\dagger}, \perp, \S$ Chunhui Wu, ${ }^{\ddagger}$ Zhiqiang Li, ${ }^{\ddagger}$ Guanghui Tian,${ }^{\ddagger}$ Jianzhong Wu ${ }^{\ddagger}$ Yang $\mathrm{He}^{*}, \stackrel{\dagger}{ }$ and Jingshan Shen ${ }^{*}, \dagger$

${ }^{\dagger}$ CAS Key Laboratory for Receptor Research, Shanghai Institute of Materia Medica, Chinese Academy of Sciences, 555 Zuchongzhi Road, Shanghai 201203, China

${ }^{\perp}$ University of Chinese Academy of Sciences, No.19A Yuquan Road, Beijing 100049, China

†Topharman Shanghai Co., Ltd., 1088 Chuansha Road, Shanghai 201209, China

\section{Context}

Analytical Data of Related Compounds

P2-P20 


\section{Compound 8}

${ }^{1} \mathrm{H}$ NMR $\left(400 \mathrm{MHz}, \mathrm{CDCl}_{3}\right) \delta 10.57(\mathrm{~s}, 1 \mathrm{H}), 7.18-7.12(\mathrm{~m}, 1 \mathrm{H}), 7.12-7.04(\mathrm{~m}, 3 \mathrm{H}), 5.42(\mathrm{~d}, J=$ $1.5 \mathrm{~Hz}, 1 \mathrm{H}), 5.26(\mathrm{~s}, 1 \mathrm{H}), 2.25(\mathrm{~s}, 3 \mathrm{H})$.

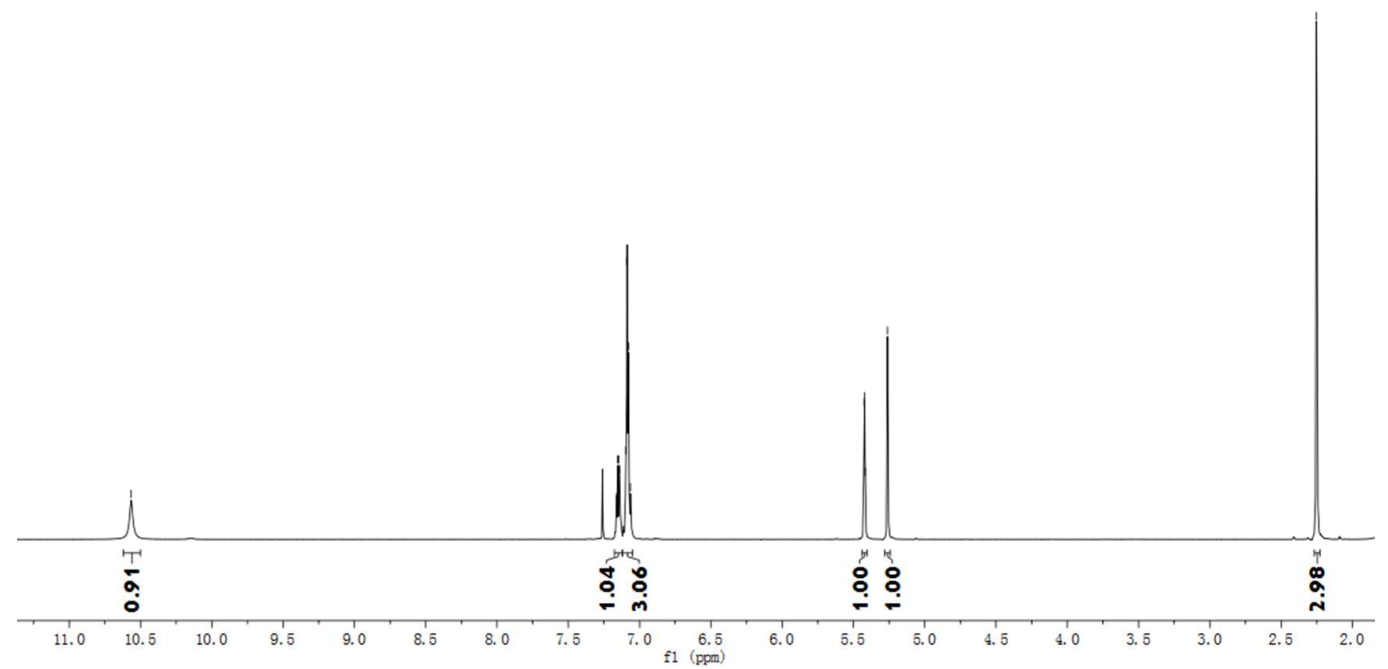




\section{Compound 9}

${ }^{1} \mathrm{H}$ NMR (400 MHz, $\left.\mathrm{CDCl}_{3}\right) \delta 7.15-7.06(\mathrm{~m}, 4 \mathrm{H}), 5.36(\mathrm{~d}, J=1.4 \mathrm{~Hz}, 1 \mathrm{H}), 5.21(\mathrm{~s}, 1 \mathrm{H}), 4.28(\mathrm{t}, J$

$=7.0 \mathrm{~Hz}, 2 \mathrm{H}), 3.66(\mathrm{t}, J=7.0 \mathrm{~Hz}, 2 \mathrm{H}), 2.23(\mathrm{~s}, 3 \mathrm{H})$.
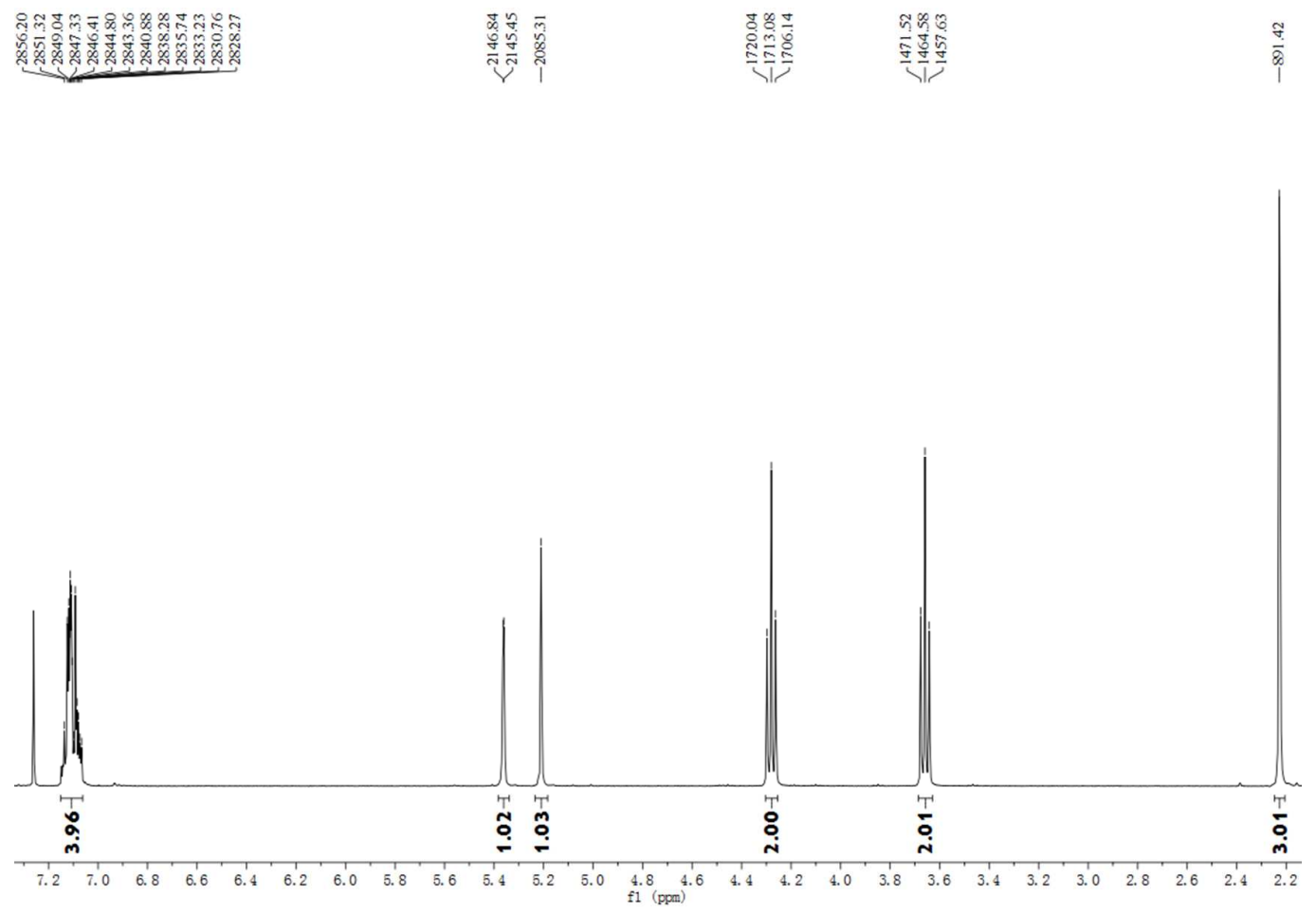


\section{Compound 11}

${ }^{1} \mathrm{H}$ NMR $\left(400 \mathrm{MHz}, \mathrm{CDCl}_{3}\right) \delta 7.34(\mathrm{t}, J=8.0 \mathrm{~Hz}, 1 \mathrm{H}), 7.14-7.02(\mathrm{~m}, 7 \mathrm{H}), 5.34(\mathrm{q}, J=1.4 \mathrm{~Hz}$, 1H), 5.19 (d, $J=0.8 \mathrm{~Hz}, 1 \mathrm{H}), 4.06$ (t, $J=7.0 \mathrm{~Hz}, 2 \mathrm{H}), 3.22$ (brt, 4H), 2.77 (t, $J=7.0 \mathrm{~Hz}, 2 \mathrm{H}), 2.72$ (brt, 4H), $2.22(\mathrm{~s}, 3 \mathrm{H})$.
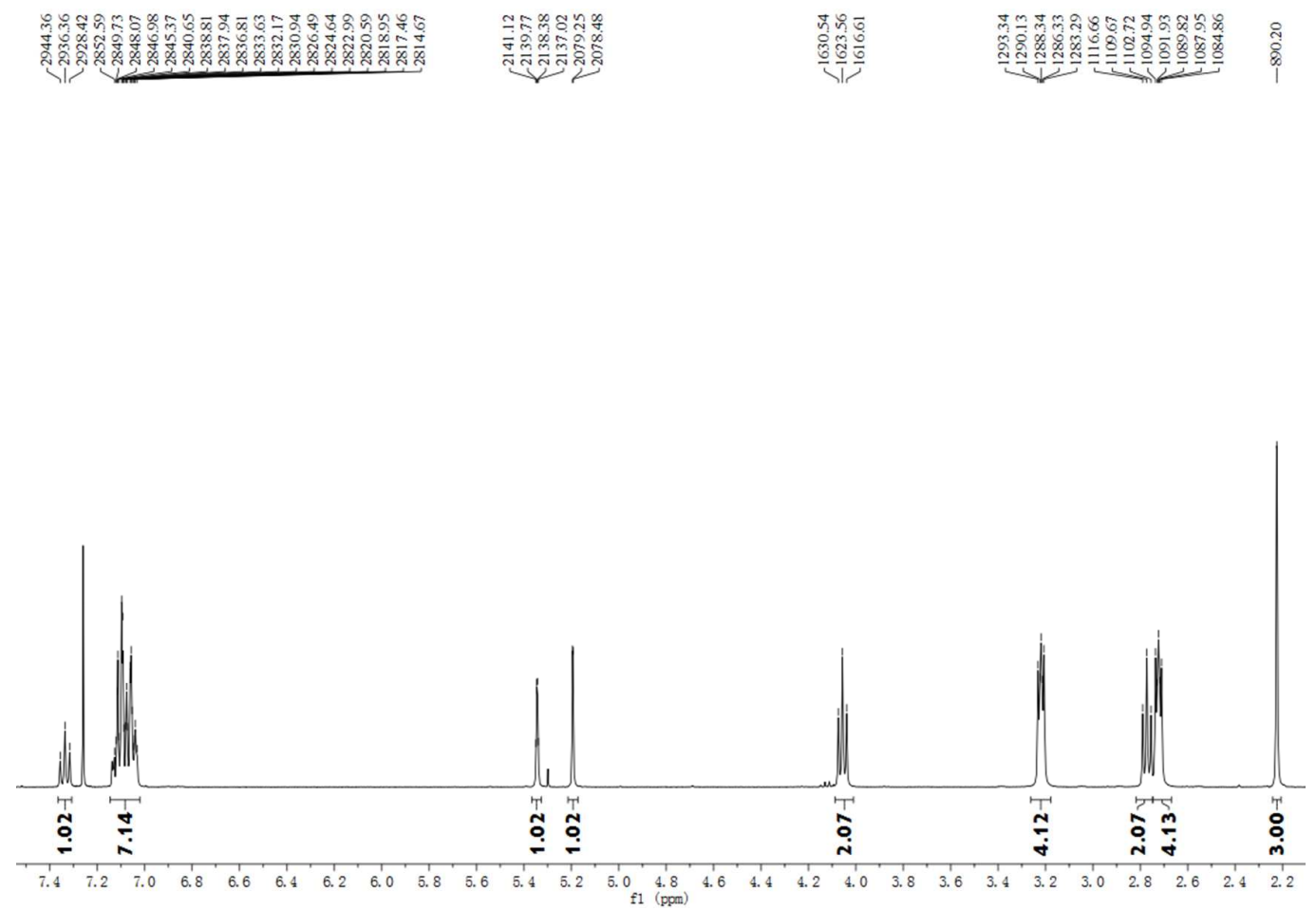
4. Compound $\mathbf{1 2}$

${ }^{1} \mathrm{H}$ NMR (400 MHz, DMSO-d $) \delta 11.80$ (brs, 1H), $7.29(\mathrm{~m}, 2 \mathrm{H}), 7.03(\mathrm{~m}, 2 \mathrm{H}), 4.48(\mathrm{q}, J=7.1 \mathrm{~Hz}$, 2H), $1.37(\mathrm{t}, J=7.1 \mathrm{~Hz}, 3 \mathrm{H})$.

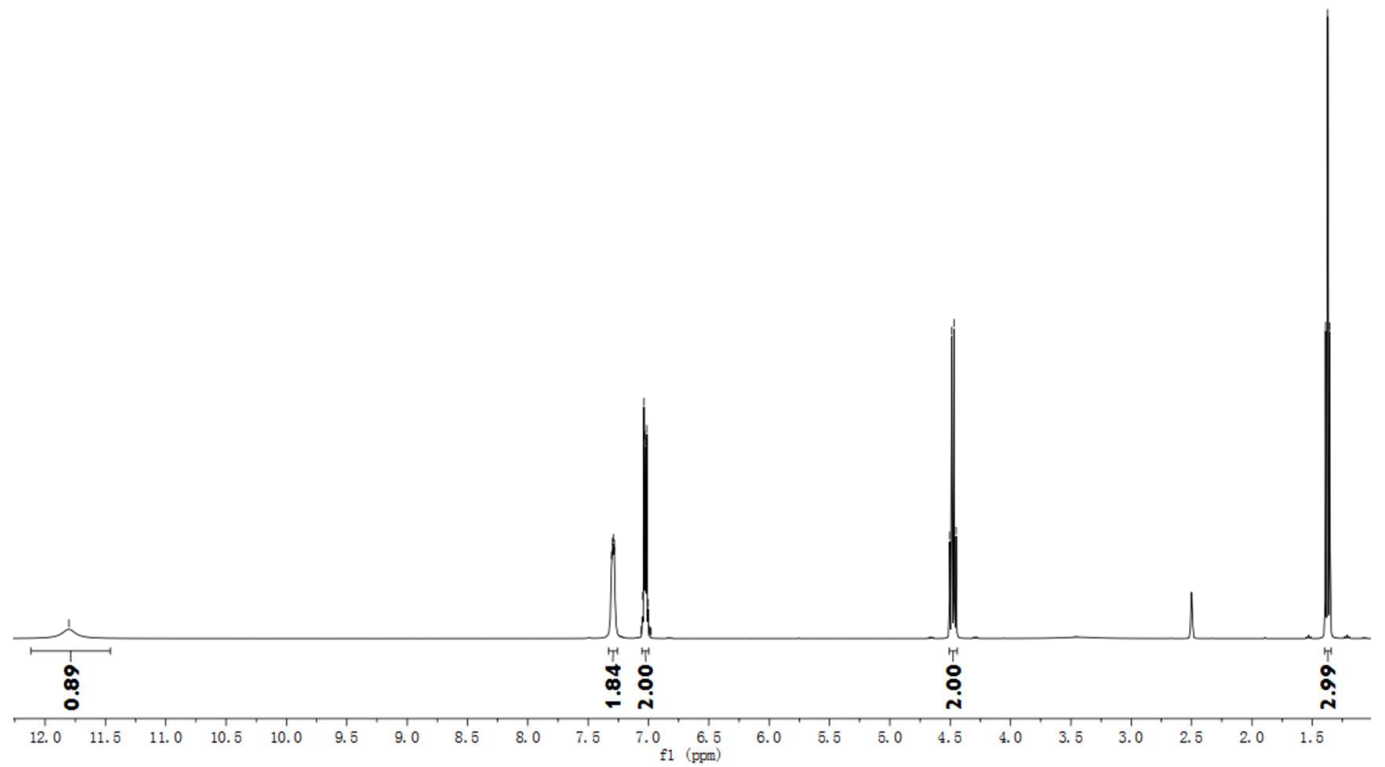




\section{Compound 13}

${ }^{1} \mathrm{H}$ NMR $\left(500 \mathrm{MHz}, \mathrm{CDCl}_{3}\right) \delta 7.52(\mathrm{~d}, J=7.6 \mathrm{~Hz}, 1 \mathrm{H}), 7.17-7.06(\mathrm{~m}, 3 \mathrm{H}), 4.58(\mathrm{q}, J=7.1 \mathrm{~Hz}$, 2H), $4.17(\mathrm{t}, J=6.6 \mathrm{~Hz}, 2 \mathrm{H}), 3.69(\mathrm{t}, J=6.6 \mathrm{~Hz}, 2 \mathrm{H}), 1.44(\mathrm{t}, J=7.1 \mathrm{~Hz}, 3 \mathrm{H})$.
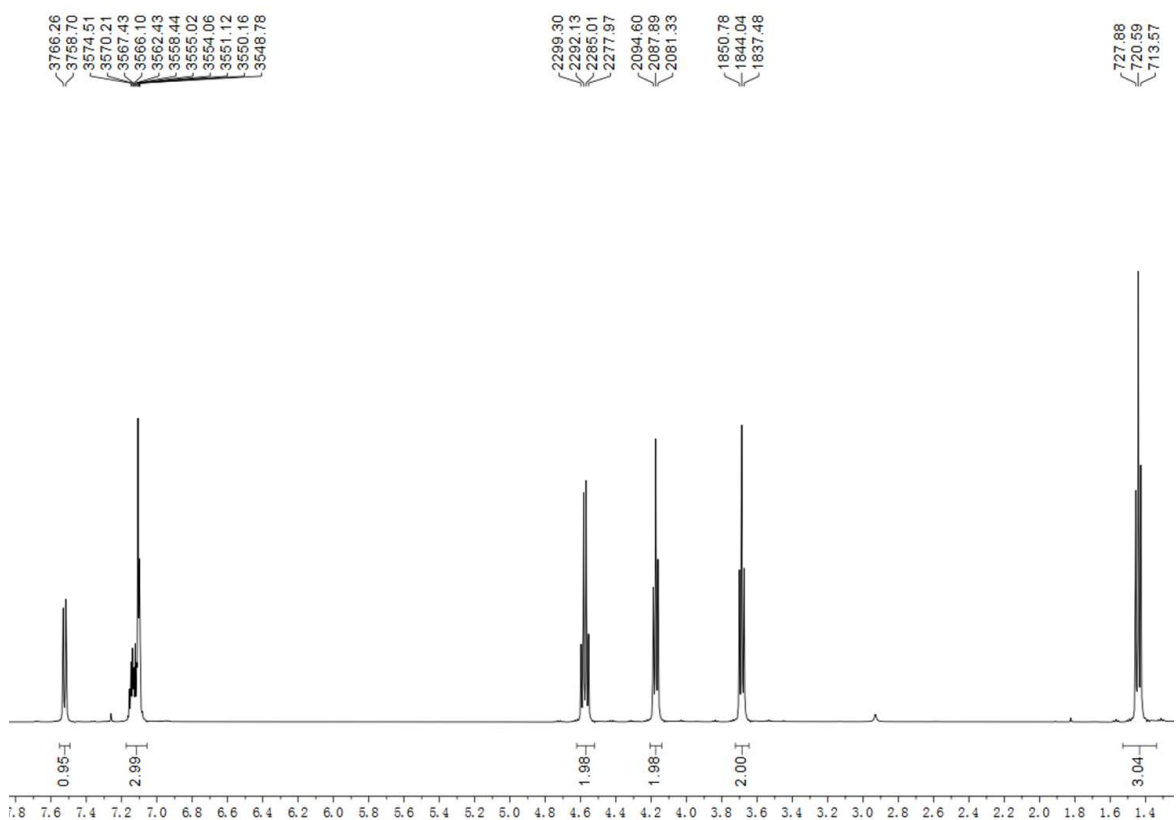

${ }^{13} \mathrm{C}$ NMR $\left(125 \mathrm{MHz}, \mathrm{CDCl}_{3}\right) \delta 156.9,140.0,133.3,121.6,120.9,117.6,107.9,66.3,43.4,41.2$, 14.6.

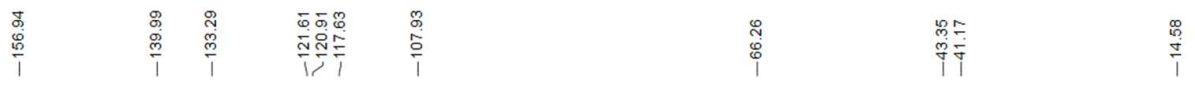
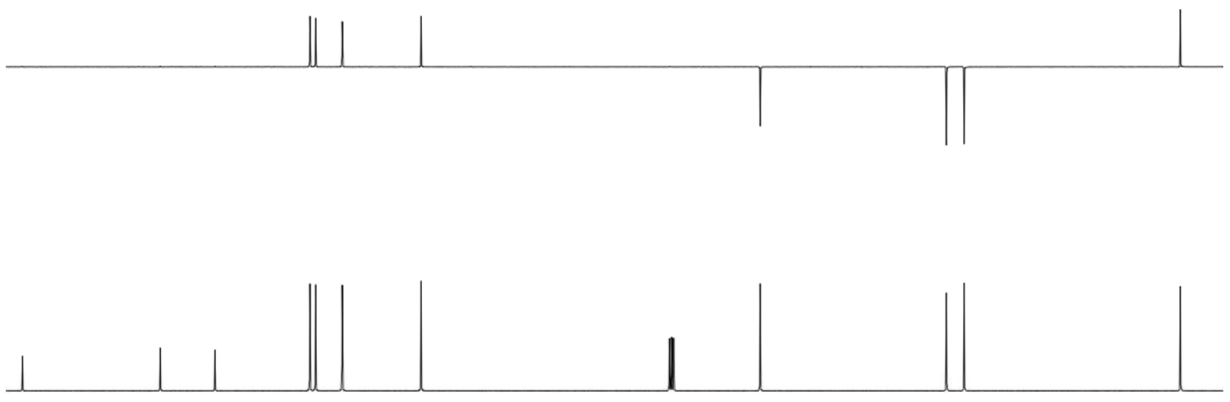

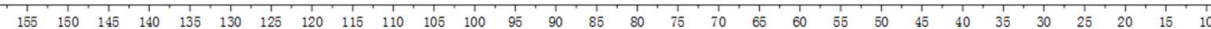


HRMS (ESI) calcd $[\mathrm{M}+\mathrm{H}]^{+}$for $\mathrm{C}_{11} \mathrm{H}_{14} \mathrm{ClN}_{2} \mathrm{O} 225.0795$, found 225.0798 .

\section{Elemental Composition Report}

Tolerance $=20.0$ PPM / DBE: $\min =-1.5, \max =50.0$

Isotope cluster parameters: Separation $=1.0$ Abundance $=1.0 \%$

Monoisotopic Mass, Even Electron lons

13 formula(e) evaluated with 1 results within limits (up to 20 closest results for each mass)

SIMM-Mass Spec

Q-Tof Ultima

20160316_ESIH_YJM_ZB_160487 133 (2.511) AM (Cen,4, 80.00, Ht,6000.0,419.21,0.70); Sm (SG, 2×1.00); Cm (116:139)

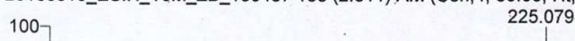

16-Mar-2016 ZZY
TOF MS ES+ $1.40 \mathrm{e} 3$

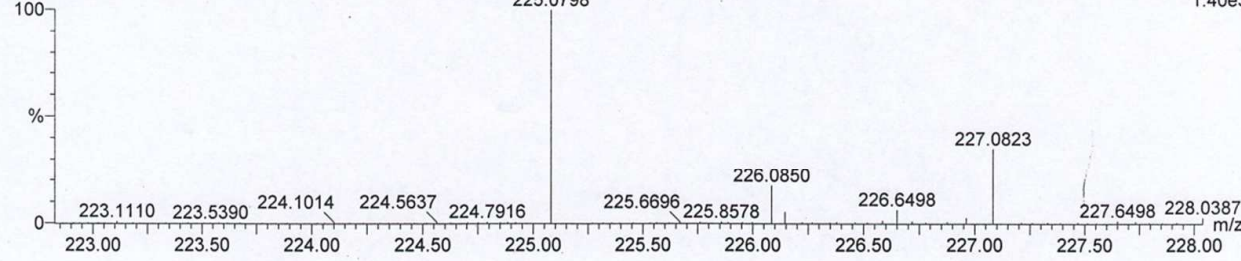

Minimum: $\quad 50.00$

Maximum: $\quad 100.00$

$200.0 \quad 20.0 \quad 50.0$

Mass

RA

Calc. Mass

$\mathrm{mDa}$

PPM

DBE

score

Formula

$225.0798 \quad 100.00$

225.0795

0.3

1.5

5.51

C11 H14 N2 O Cl 
HPLC: retention time of $16.0 \mathrm{~min}, 97.5 \%$ pure.

1/13/2016 8:49:42 AM Page 1/1

\section{LabSolutions Analysis Report}

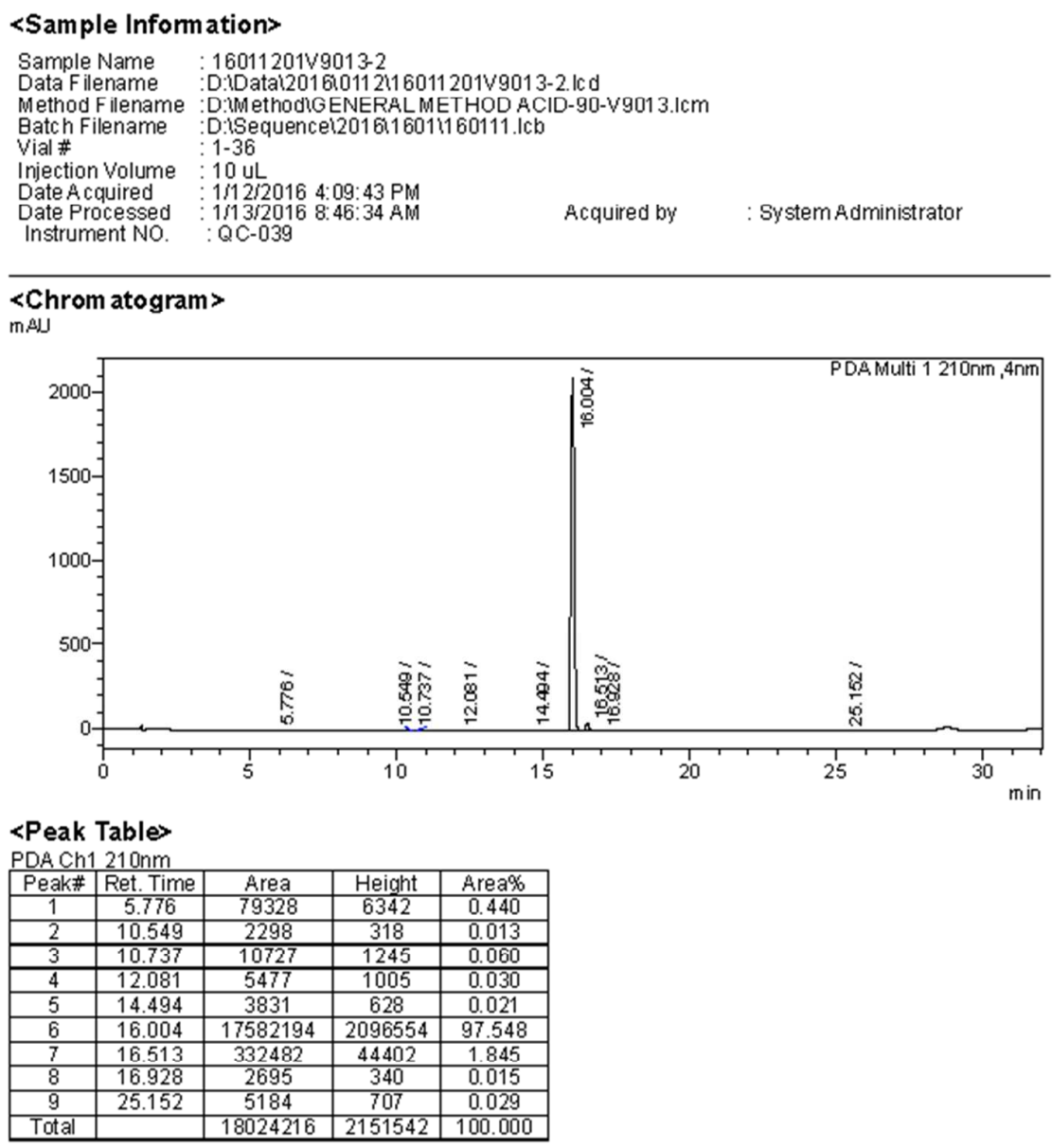

D:Satai201610112/16011201v9013-2.Icd 


\section{Compound 14}

${ }^{1} \mathrm{H}$ NMR $\left(500 \mathrm{MHz}, \mathrm{CDCl}_{3}\right) \delta 7.56(\mathrm{~m}, 1 \mathrm{H}), 7.32(\mathrm{t}, J=8.0 \mathrm{~Hz}, 1 \mathrm{H}), 7.21-7.12(\mathrm{~m}, 3 \mathrm{H}), 7.10$ (brt, $J=1.9 \mathrm{~Hz}, 1 \mathrm{H}), 7.07(\mathrm{~d}, J=7.7 \mathrm{~Hz}, 1 \mathrm{H}), 7.02(\mathrm{dd}, J=8.4,2.4 \mathrm{~Hz}, 1 \mathrm{H}), 4.62(\mathrm{q}, J=7.1 \mathrm{~Hz}, 2 \mathrm{H})$, $4.11(\mathrm{t}, J=6.8 \mathrm{~Hz}, 2 \mathrm{H}), 3.19$ (brt, $J=5.0 \mathrm{~Hz}, 4 \mathrm{H}), 2.75(\mathrm{t}, J=6.9 \mathrm{~Hz}, 2 \mathrm{H}), 2.66(\mathrm{t}, J=5.0 \mathrm{~Hz}$, 4H), 1.49 (t, $J=7.1 \mathrm{~Hz}, 3 \mathrm{H})$.

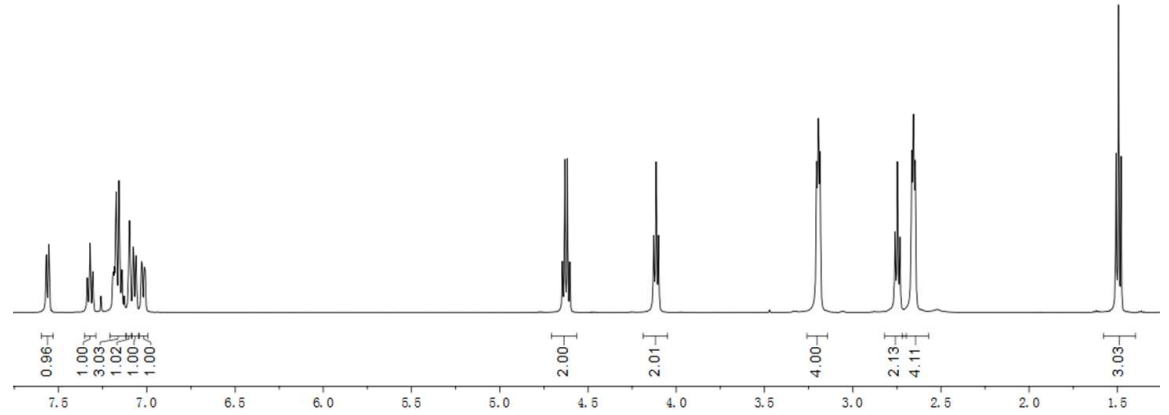

${ }^{13} \mathrm{C}$ NMR $\left(125 \mathrm{MHz}, \mathrm{CDCl}_{3}\right) \delta 157.4,151.3,140.3,133.6,131.4$ (q, $\left.J=31.8 \mathrm{~Hz}\right), 129.6,124.4$ (q, $J=272.5 \mathrm{~Hz}), 121.5,120.8,118.7,117.7,115.9(\mathrm{q}, J=3.9 \mathrm{~Hz}), 112.2(\mathrm{q}, J=3.8 \mathrm{~Hz}), 108.1,66.2$, $56.4,53.1 \times 2,48.6 \times 2,39.7,14.9$.
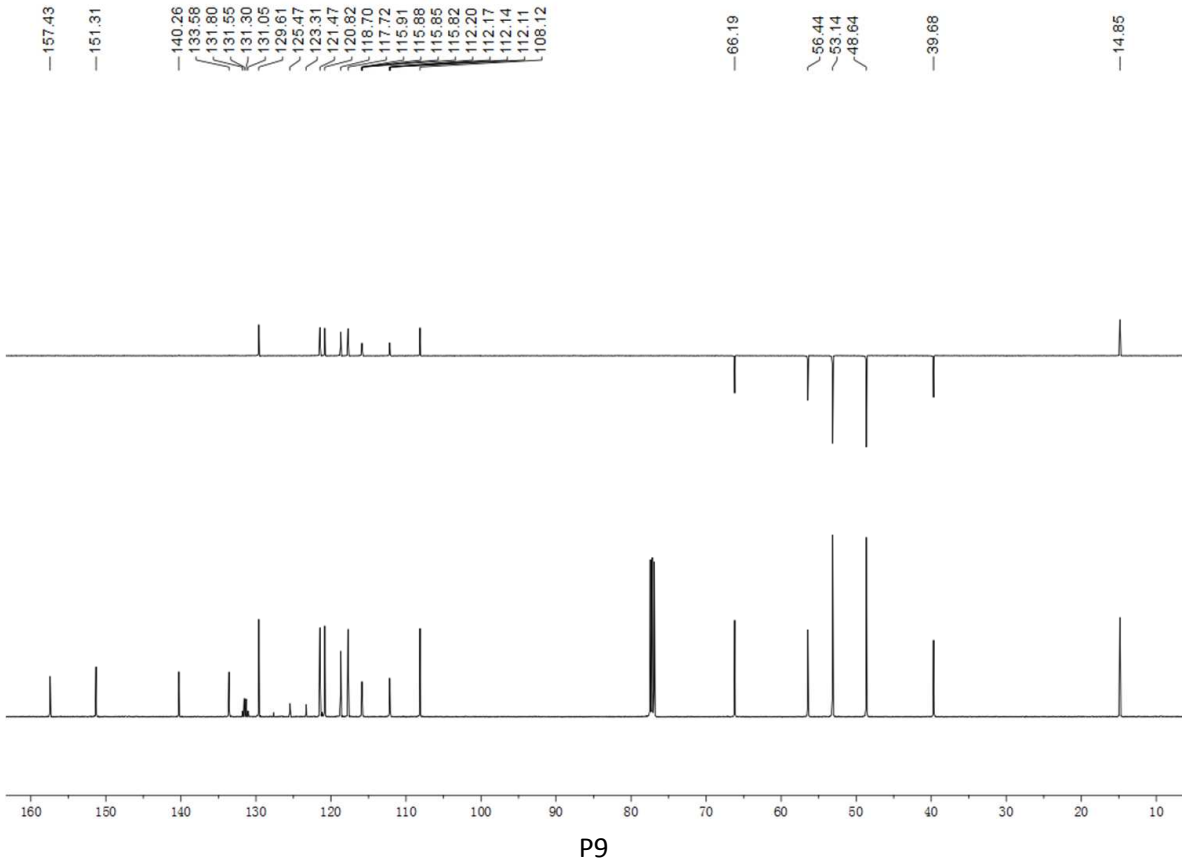
HRMS (ESI) calcd [M + H] for $\mathrm{C}_{22} \mathrm{H}_{26} \mathrm{~F}_{3} \mathrm{~N}_{4} \mathrm{O}$ 419.2059, found 419.2071.

Elemental Composition Report

Page 1

Tolerance $=20.0$ PPM / DBE: $\min =-1.5, \max =50.0$

Isotope cluster parameters: Separation $=1.0$ Abundance $=1.0 \%$

Monoisotopic Mass, Even Electron Ions

44 formula(e) evaluated with 1 results within limits (up to 20 closest results for each mass)

SIMM-Mass Spec

Q-Tof Ultima

16-Mar-2016

$545 \mathrm{C}$

20160316 ESIH_YJM_ZB_160486 1040 (19.607) AM (Cen,4, 80.00, Ht,6000.0,362.93,0.70); Sm (SG, 2x1.00); Cm (1004:1042)

100

419.2071

ZZY
TOF MS ES+
$1.77 \mathrm{e} 3$

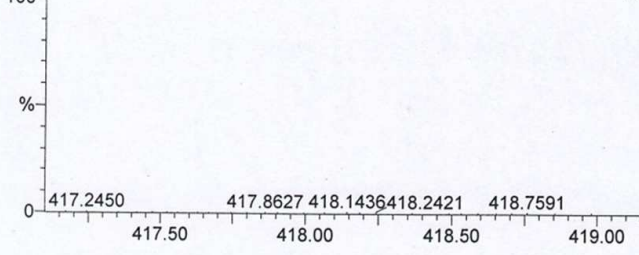

Minimum: $\quad 50.00$

Maximum: $\quad 100.00$

$200.0 \quad 20.0$

-1.5
50.0

Mass RA Calc. Mass mDa PPM DBE

BE Score Formula

$\begin{array}{lllll}419.2071 & 100.00 & 419.2059 & 1.2 & 2.9\end{array}$

10.51

$\begin{array}{lllll}\mathrm{C} 22 & \mathrm{H} 26 & \mathrm{~N} 4 & \mathrm{O} & \mathrm{F} 3\end{array}$ 
HPLC: retention time of $12.4 \mathrm{~min}, 95 \%$ pure.
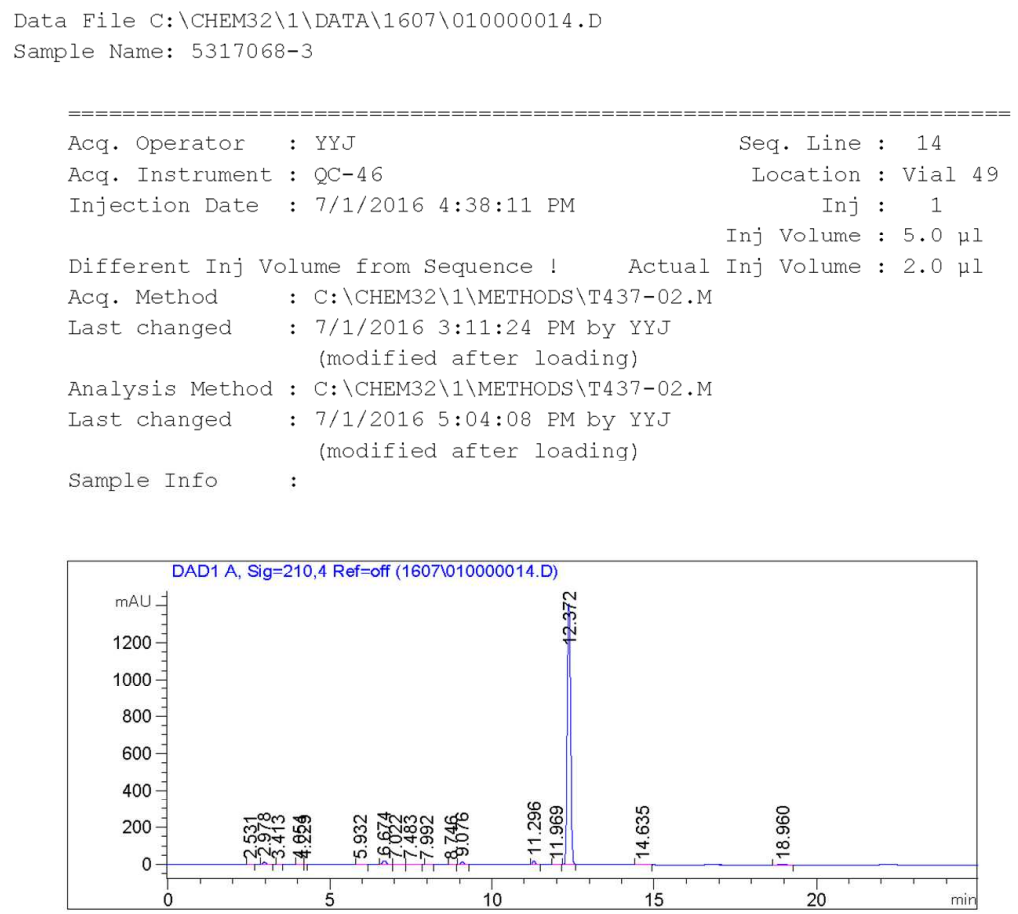

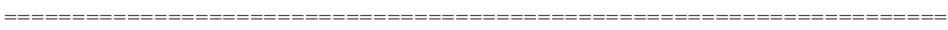

Area Percent Report

$\begin{array}{llll}\text { Sorted By } & : & \text { Signal } & \\ \text { Multiplier: } & & : & 1.0000 \\ \text { Dilution: } & & : & 1.0000\end{array}$

Use Multiplier \& Dilution Factor with ISTDs

Signal 1: DAD1 A, Sig=210,4 Ref=off

\begin{tabular}{|c|c|c|c|c|c|c|}
\hline $\begin{array}{c}\text { eak } \\
\text { \# }\end{array}$ & $\begin{array}{c}\text { RetTime } \\
\text { [min] }\end{array}$ & Type & $\begin{array}{l}\text { Width } \\
\text { [min] }\end{array}$ & $\begin{array}{c}\text { Area } \\
{\left[\mathrm{mAU}{ }^{*} \mathrm{~s}\right]}\end{array}$ & $\begin{array}{l}\text { Height } \\
\text { [MAU] }\end{array}$ & $\begin{array}{c}\text { Area } \\
\text { 응 }\end{array}$ \\
\hline & & & & & & \\
\hline 1 & 2.531 & $\mathrm{BB}$ & 0.0900 & 3.40081 & $5.83088 e-1$ & 0.0316 \\
\hline 2 & 2.978 & $\mathrm{BB}$ & 0.1081 & 82.08157 & 11.95681 & 0.7616 \\
\hline 3 & 3.413 & $\mathrm{BB}$ & 0.0764 & 1.90508 & $3.56141 e-1$ & 0.0177 \\
\hline 4 & & BV & 0.1154 & 13.77289 & 1.92598 & 0.1278 \\
\hline 5 & 4.229 & $\mathrm{VB}$ & 0.0172 & $4.25911 e-2$ & $6.88998 e-2$ & $3.952 e-4$ \\
\hline 6 & 5.932 & $\mathrm{BB}$ & 0.1381 & 10.60030 & 1.18750 & 0.0983 \\
\hline 7 & 6.674 & BV & 0.1192 & 143.67491 & 19.66291 & 1.3330 \\
\hline 8 & 7.022 & VV & 0.1448 & 9.96235 & $9.31422 e-1$ & 0.0924 \\
\hline 9 & 7.483 & VV & 0.1237 & 14.52652 & 1.89017 & 0.1348 \\
\hline 10 & 7.992 & $\mathrm{BB}$ & 0.0892 & 2.42155 & $3.86073 e-1$ & 0.0225 \\
\hline 11 & 8.746 & $\mathrm{BB}$ & 0.0863 & 1.85102 & $3.16019 e-1$ & 0.0172 \\
\hline 12 & 9.076 & $\mathrm{BB}$ & 0.1056 & 89.50344 & 13.82115 & 0.8304 \\
\hline 13 & 11.296 & $\mathrm{BB}$ & 0.0993 & 135.85141 & 22.24079 & 1.2604 \\
\hline 14 & 11.969 & $\mathrm{BB}$ & 0.0979 & 7.64410 & 1.20714 & 0.0709 \\
\hline
\end{tabular}




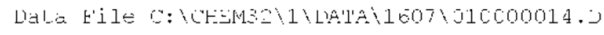

simple Nax: $\equiv 317068-3$

\begin{tabular}{|c|c|c|c|c|c|c|}
\hline $\begin{array}{l}\text { Eed: } \\
\text { H }\end{array}$ & $\begin{array}{c}\text { ReLTime } \\
\text { [mir }]\end{array}$ & Type & $\begin{array}{l}\text { ricich } \\
\text { [minin] }\end{array}$ & $\begin{array}{c}A_{\perp} \in A \\
{\left[\mathrm{~m} \cdot \mathrm{L}^{-*} \mathrm{~g}\right]}\end{array}$ & $\begin{array}{l}F \in i g h l \\
\left.\left[m^{\prime} \bar{s}^{\prime} I\right]\right]\end{array}$ & $\begin{array}{c}\text { Ancea } \\
y\end{array}$ \\
\hline \multicolumn{7}{|c|}{--- |-------|----| } \\
\hline $1 b$ & $1 \because 3 \because 2$ & & $\therefore 1186$ & 1.2202304 & 2411.0303 & 94,9442 \\
\hline 16 & 14.635 & $L^{\prime \prime}$ & 0.1500 & - ¿. 43159 & $9.300300-$ & 0960 \\
\hline 17 & 12.960 & & c. $=10 \%$ & -7.19332 & $1.0 \div 120$ & $.159=$ \\
\hline
\end{tabular}

l'wlals: $\quad 1.0778204 \quad-490.3779$

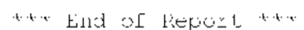




\section{Compound 1}

${ }^{1} \mathrm{H}$ NMR $\left(400 \mathrm{MHz}\right.$, DMSO- $\left.d_{6}\right) \delta 10.83(\mathrm{~s}, 1 \mathrm{H}), 7.40(\mathrm{t}, J=8.0 \mathrm{~Hz}, 1 \mathrm{H}), 7.20(\mathrm{dd}, J=8.4 \mathrm{~Hz}, 2.4$

$\mathrm{Hz}, 1 \mathrm{H}), 7.12-7.17(\mathrm{~m}, 2 \mathrm{H}), 7.05(\mathrm{~d}, J=7.6 \mathrm{~Hz}, 1 \mathrm{H}), 6.94-7.02(\mathrm{~m}, 3 \mathrm{H}), 3.94(\mathrm{t}, J=6.6 \mathrm{~Hz}, 2 \mathrm{H})$, 3.17 (brt, $4 \mathrm{H}), 2.58-2.65(\mathrm{~m}, 6 \mathrm{H})$.

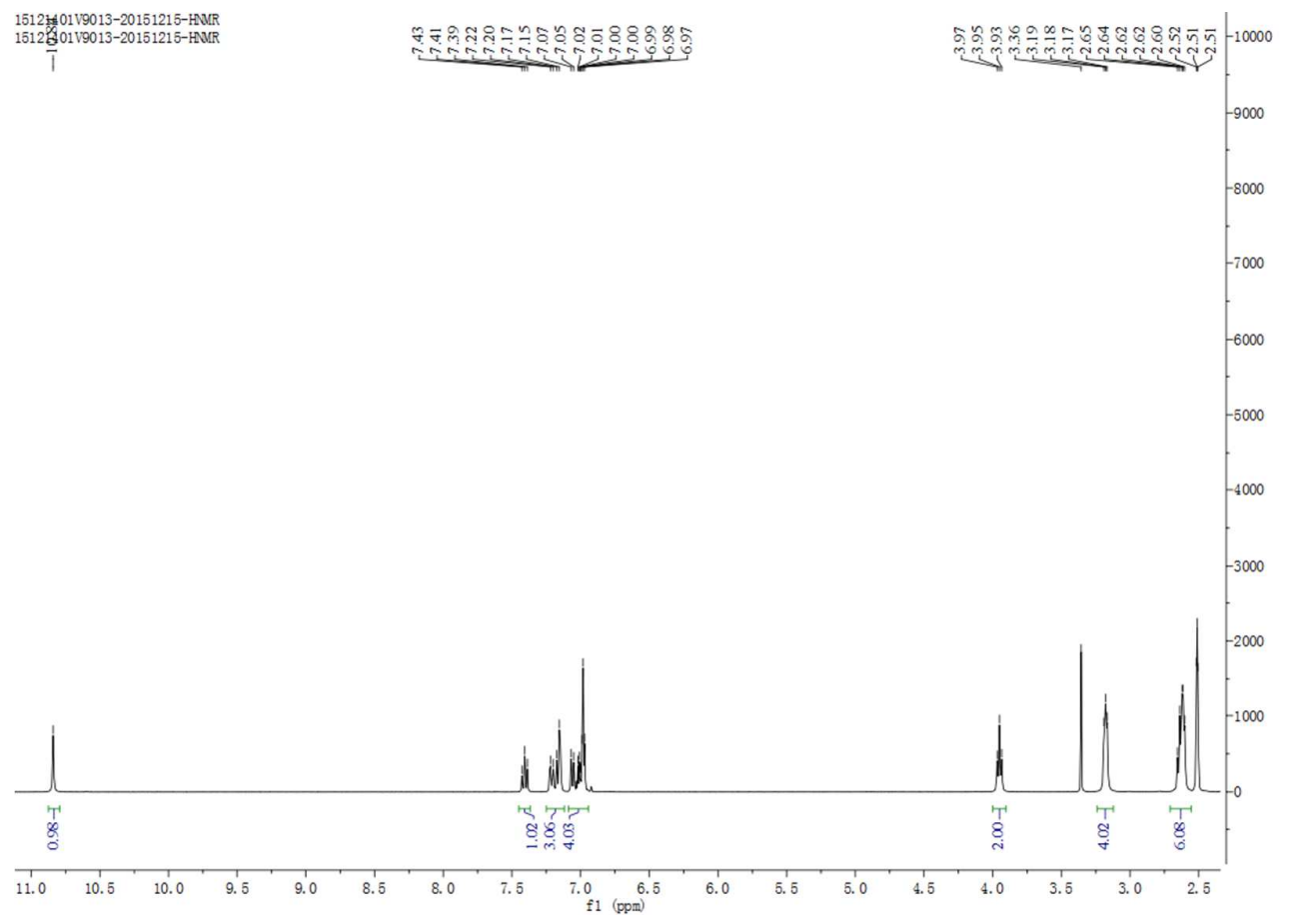


HPLC: retention time of 9.06 min, $99.9 \%$ pure.
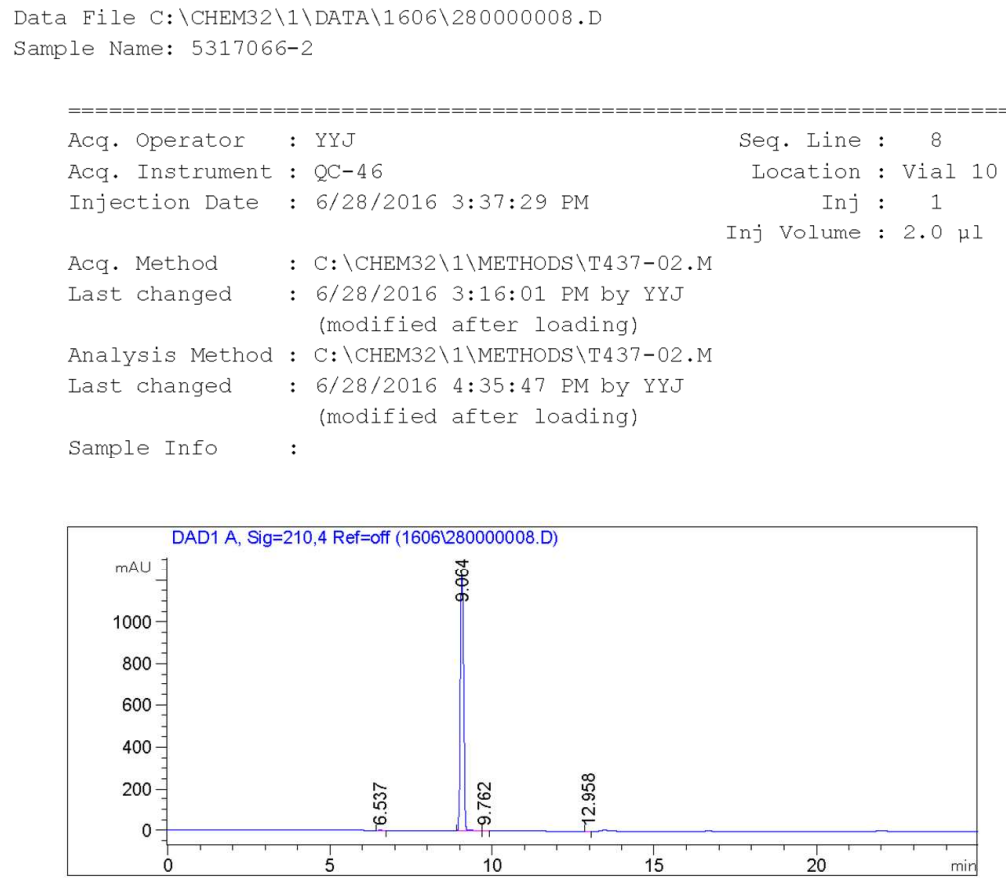

Area Percent Report

\begin{tabular}{|c|c|c|c|}
\hline Sorted By & : & Signal & \\
\hline Multiplier: & & : & 1.0000 \\
\hline Dilution: & & $:$ & 1.0000 \\
\hline
\end{tabular}

Signal 1: DAD1 A, Sig=210,4 Ref=off

\begin{tabular}{|c|c|c|c|c|c|c|}
\hline $\begin{array}{c}\text { Peak } \\
\text { \# }\end{array}$ & $\begin{array}{c}\text { RetTime } \\
\text { [min] }\end{array}$ & Type & $\begin{array}{l}\text { Width } \\
\text { [min] }\end{array}$ & $\begin{array}{c}\text { Area } \\
{\left[\mathrm{mAU}^{*} \mathrm{~S}\right]}\end{array}$ & $\begin{array}{l}\text { Height } \\
\text { [mAU] }\end{array}$ & $\begin{array}{c}\text { Area } \\
\frac{8}{8}\end{array}$ \\
\hline \multicolumn{7}{|c|}{$----\mid-------$} \\
\hline 1 & 6.537 & $\mathrm{BB}$ & 0.1044 & 3.56915 & $5.31024 e-1$ & 0.0403 \\
\hline 2 & 9.064 & BV & 0.1186 & 8838.96973 & 1249.10681 & 99.9071 \\
\hline 3 & 9.762 & VB & 0.1086 & 4.13273 & $5.43891 e-1$ & 0.0467 \\
\hline 4 & 12.958 & BV & 0.0737 & $5.14206 e-1$ & $9.72614 e-2$ & $5.812 \mathrm{e}-3$ \\
\hline t & & & & 8847.18581 & .27899 & \\
\hline
\end{tabular}

$* * *$ End of Report *** 
8. Compound 15

${ }^{1} \mathrm{H}$ NMR $\left(500 \mathrm{MHz}, \mathrm{CDCl}_{3}\right) \delta 7.49(\mathrm{~d}, J=7.8 \mathrm{~Hz}, 2 \mathrm{H}), 7.13(\mathrm{t}, J=7.8 \mathrm{~Hz}, 2 \mathrm{H}), 7.06(\mathrm{t}, J=7.6$

$\mathrm{Hz}, 2 \mathrm{H}), 6.88$ (d, $J=7.8 \mathrm{~Hz}, 2 \mathrm{H}), 4.25$ (brs, 4H), 4.24 (q, $J=7.1 \mathrm{~Hz}, 4 \mathrm{H}), 1.12$ (t, $J=7.1 \mathrm{~Hz}, 6 \mathrm{H})$.
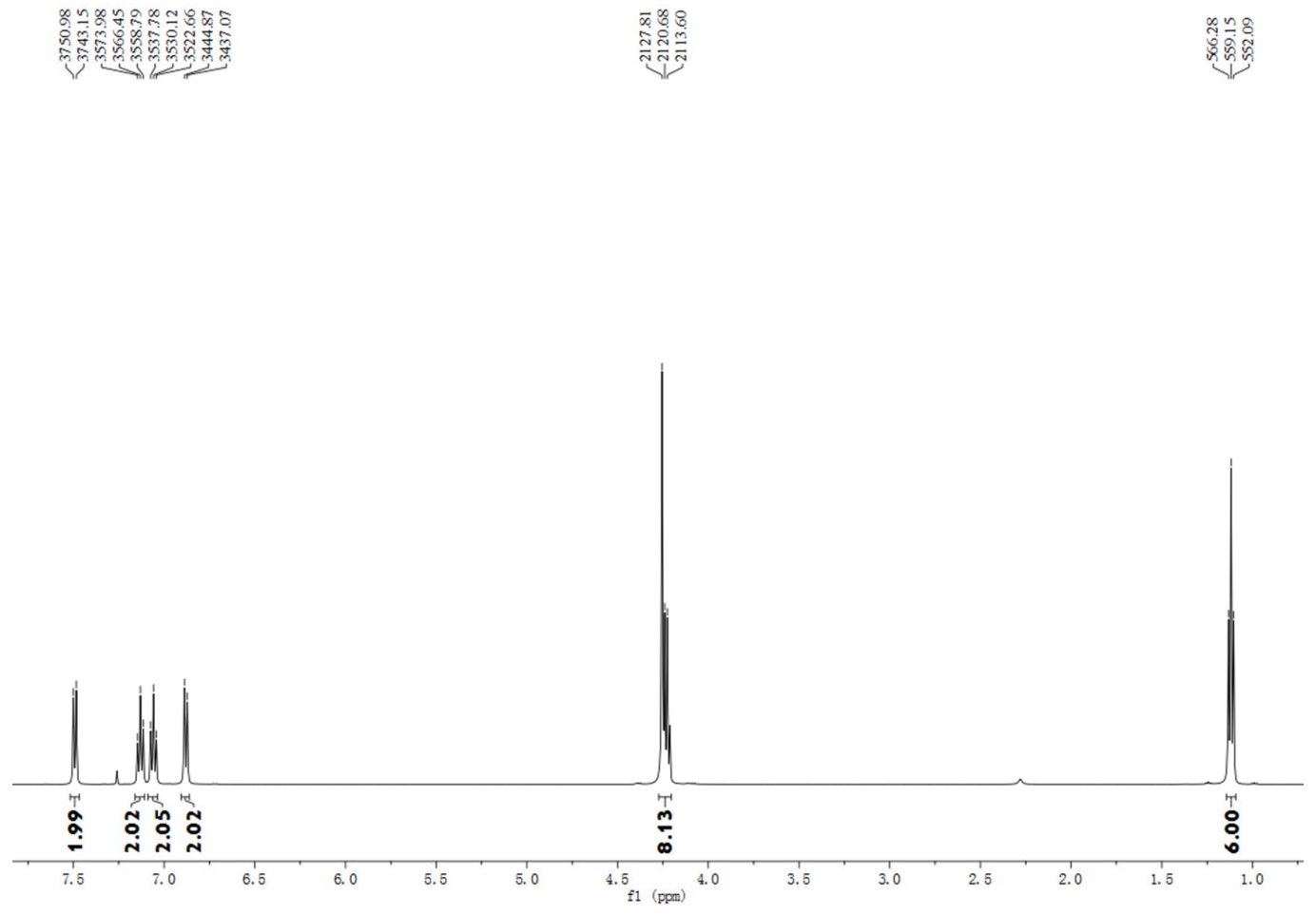

${ }^{13} \mathrm{C}$ NMR $\left(125 \mathrm{MHz}, \mathrm{CDCl}_{3}\right) \delta 157.1,140.3,133.2,121.8,121.1,117.9,107.3,66.2,40.6,14.2$.
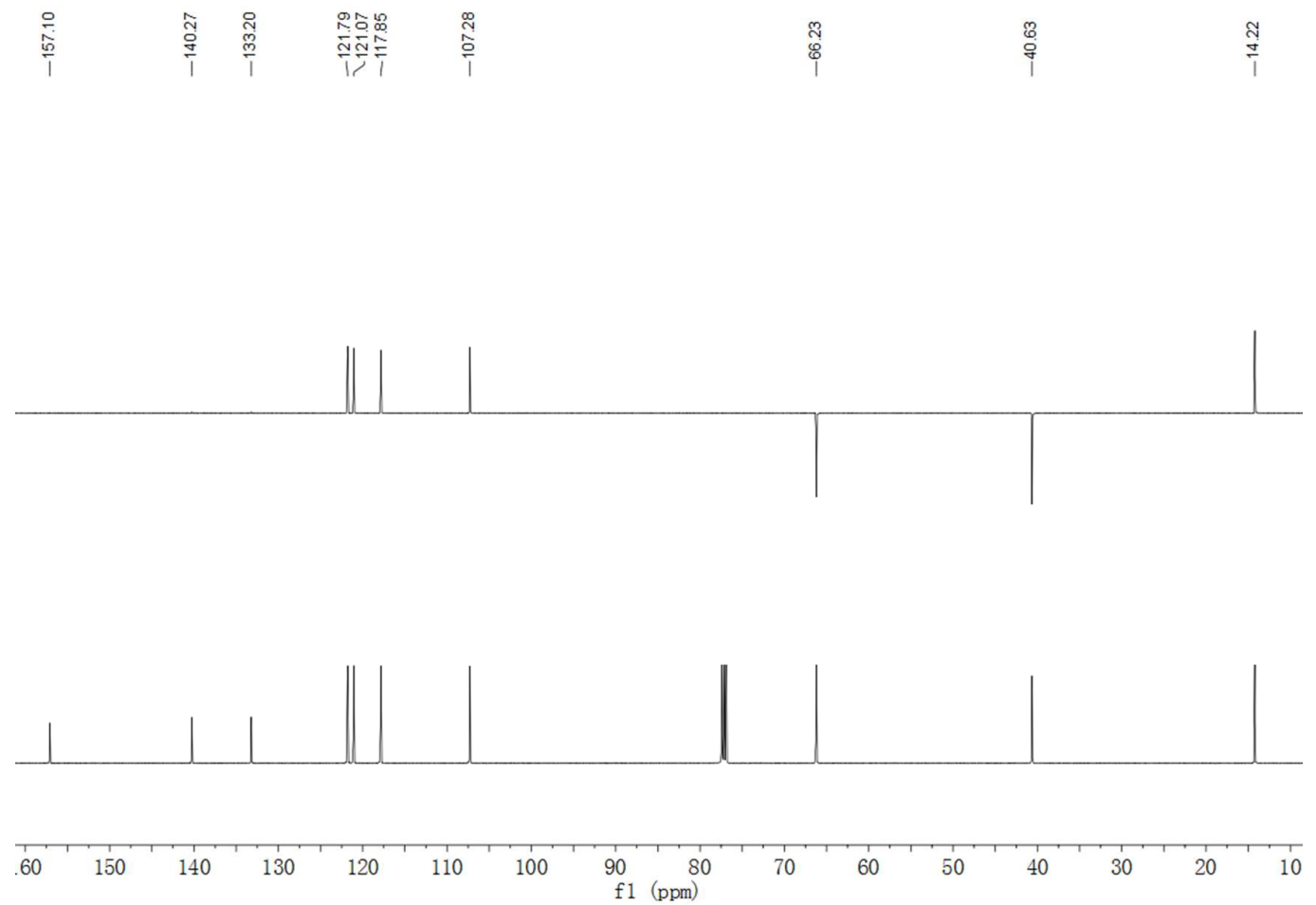

$\mathrm{P} 15$ 
HRMS (ESI) calcd [M + H] for $\mathrm{C}_{20} \mathrm{H}_{23} \mathrm{~N}_{4} \mathrm{O}_{2}$ 351.1821, found 351.1814.

Elemental Composition Report

Page 1

Single Mass Analysis

Tolerance $=8.0$ PPM / DBE: $\min =-1.5, \max =50.0$

Element prediction: Off

Number of isotope peaks used for $\mathrm{i}-\mathrm{FIT}=3$

Monoisotopic Mass, Even Electron lons

285 formula(e) evaluated with 2 results within limits (up to 50 closest results for each mass)

Elements Used:

$\begin{array}{llll}C: 5-80 & \text { H: } 2-120 \quad \mathrm{~N}: 0-4 & \text { O: } 0-30\end{array}$

V9013-2C

LCT PXE KE324

24-Mar-2016

V9013-2C_0324 32 (0.687) AM2 (Ar, 13500.0,0.00,0.70); ABS; Cm (24:32)

$3.90 \mathrm{e}+003$

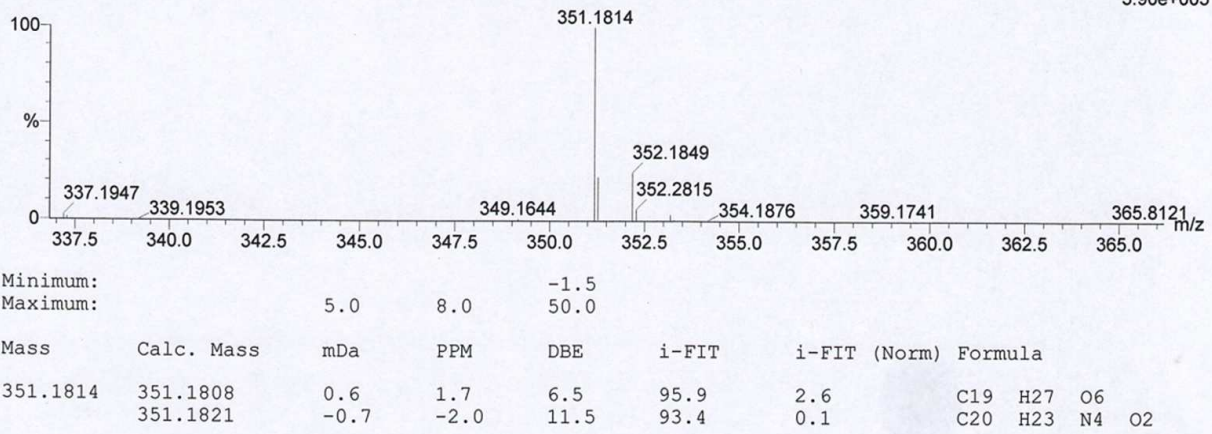




\section{Compound 16}

${ }^{1} \mathrm{H}$ NMR (500 MHz, $\left.\mathrm{CDCl}_{3}\right) \delta 7.56-7.42(\mathrm{~m}, 1 \mathrm{H}), 7.20-7.05(\mathrm{~m}, 3 \mathrm{H}), 4.59(\mathrm{q}, J=7.1 \mathrm{~Hz}, 2 \mathrm{H})$, $4.27(\mathrm{t}, J=7.1 \mathrm{~Hz}, 2 \mathrm{H}), 3.54(\mathrm{t}, J=7.1 \mathrm{~Hz}, 2 \mathrm{H}), 1.46(\mathrm{t}, J=7.1 \mathrm{~Hz}, 3 \mathrm{H})$.
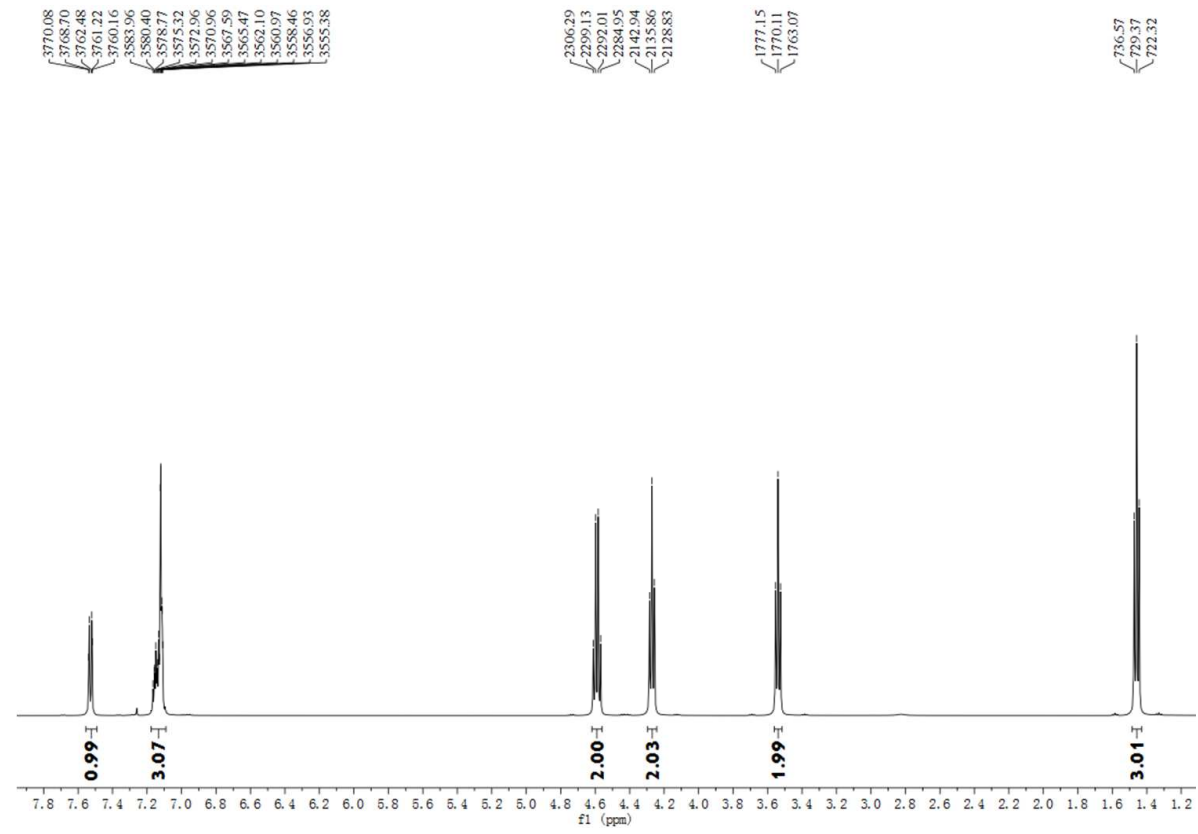

${ }^{13} \mathrm{C}$ NMR $\left(125 \mathrm{MHz}, \mathrm{CDCl}_{3}\right) \delta 156.9,140.0,133.1,121.7,121.0,117.7,107.9,66.3,43.3,28.3$, 14.7

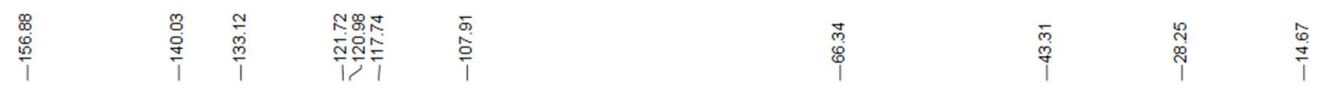
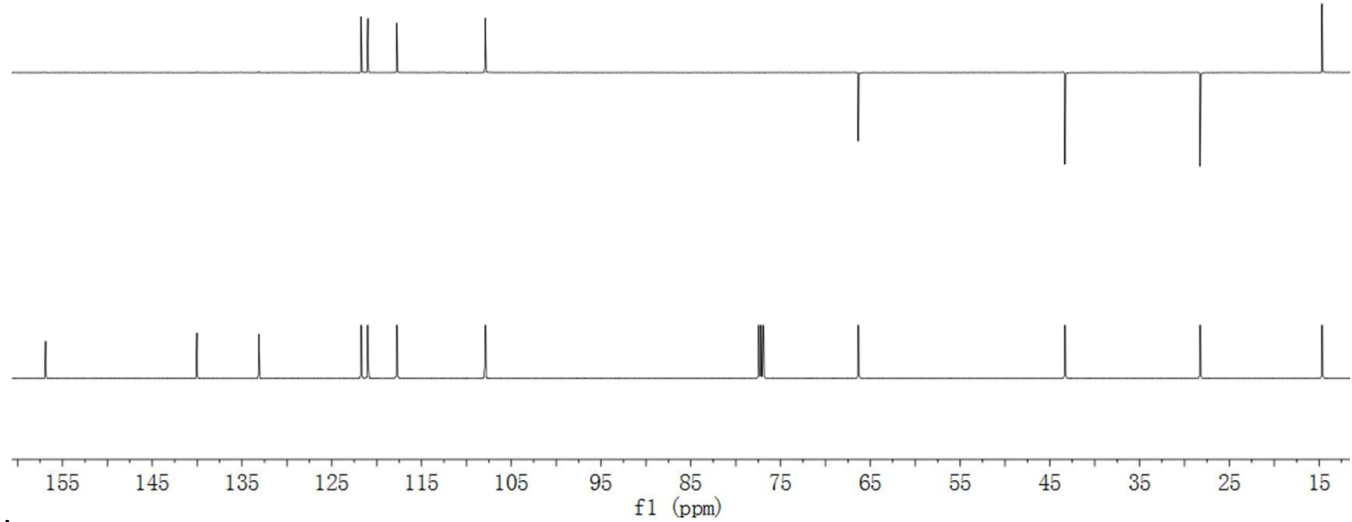
HRMS (ESI) calcd $[\mathrm{M}+\mathrm{H}]^{+}$for $\mathrm{C}_{11} \mathrm{H}_{14} \mathrm{BrN}_{2} \mathrm{O} 269.0289$, found 269.0286.

Elemental Composition Report

Page 1

Single Mass Analysis

Tolerance $=3.0$ PPM / DBE: $\min =-1.5, \max =50.0$
Element prediction: Off

Number of isotope peaks used for $\mathrm{i}-\mathrm{FIT}=3$

Monoisotopic Mass, Even Electron lons

174 formula(e) evaluated with 1 results within limits (up to 50 closest results for each mass)

Elements Used:

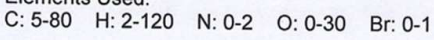

V9013-2B

LCT PXE KE324

4-Mar-2016

V9013-2B_0324 32 (0.687) AM2 (Ar, 10000.0,0.00,1.00); ABS; Cm (28:46)

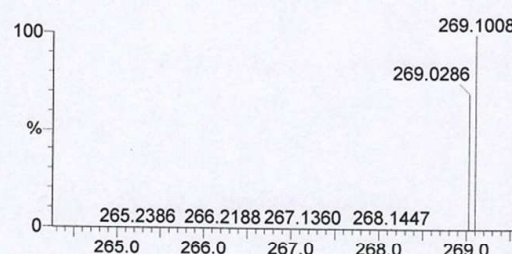

$269.1008 \quad 271.0993$

$1:$ TOF MS ES+
$4.44 \mathrm{e}+003$

$\begin{array}{lll}265.0 & 266.0 & 267\end{array}$
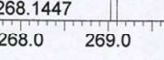

Minimum:

Maximum:

5.0

3.0

271.0265

Mass

Calc. Mass mDa

PPM

-1.5
50.0

269.0286

269.0289

$-0.3$

$-1.1$

5.5

i-FIT

i-FIT (Norm) Formula

$270.1033 \quad 272.1017$

$\begin{array}{lllllllll} & & & 273.0305 & 273.6216 & 275.2822 & 276.2882 & \\ 270.0 & 271.0 & 272.0 & 273.0 & 274.0 & 275.0 & 276.0 & 277.0\end{array}$

$-1.5$

E

154.1

0.0

$\begin{array}{lllll}\mathrm{C} 11 & \mathrm{H} 14 & \mathrm{~N} 2 & \mathrm{O} & \mathrm{Br}\end{array}$ 
10. Compound 17

${ }^{1} \mathrm{H}$ NMR $\left(400 \mathrm{MHz}, \mathrm{CDCl}_{3}\right) \delta 7.54(\mathrm{dd}, J=7.1,1.8 \mathrm{~Hz}, 1 \mathrm{H}), 7.44(\mathrm{dd}, J=7.1,1.8 \mathrm{~Hz}, 1 \mathrm{H}), 7.24-$ $7.10(\mathrm{~m}, 2 \mathrm{H}), 7.01(\mathrm{dd}, J=16.2,9.5 \mathrm{~Hz}, 1 \mathrm{H}), 5.51(\mathrm{dd}, J=16.2,0.8 \mathrm{~Hz}, 1 \mathrm{H}), 5.05-4.94(\mathrm{~m}, 1 \mathrm{H})$, $4.63(\mathrm{q}, J=7.1 \mathrm{~Hz}, 2 \mathrm{H}), 1.50(\mathrm{t}, J=7.1 \mathrm{~Hz}, 3 \mathrm{H})$.
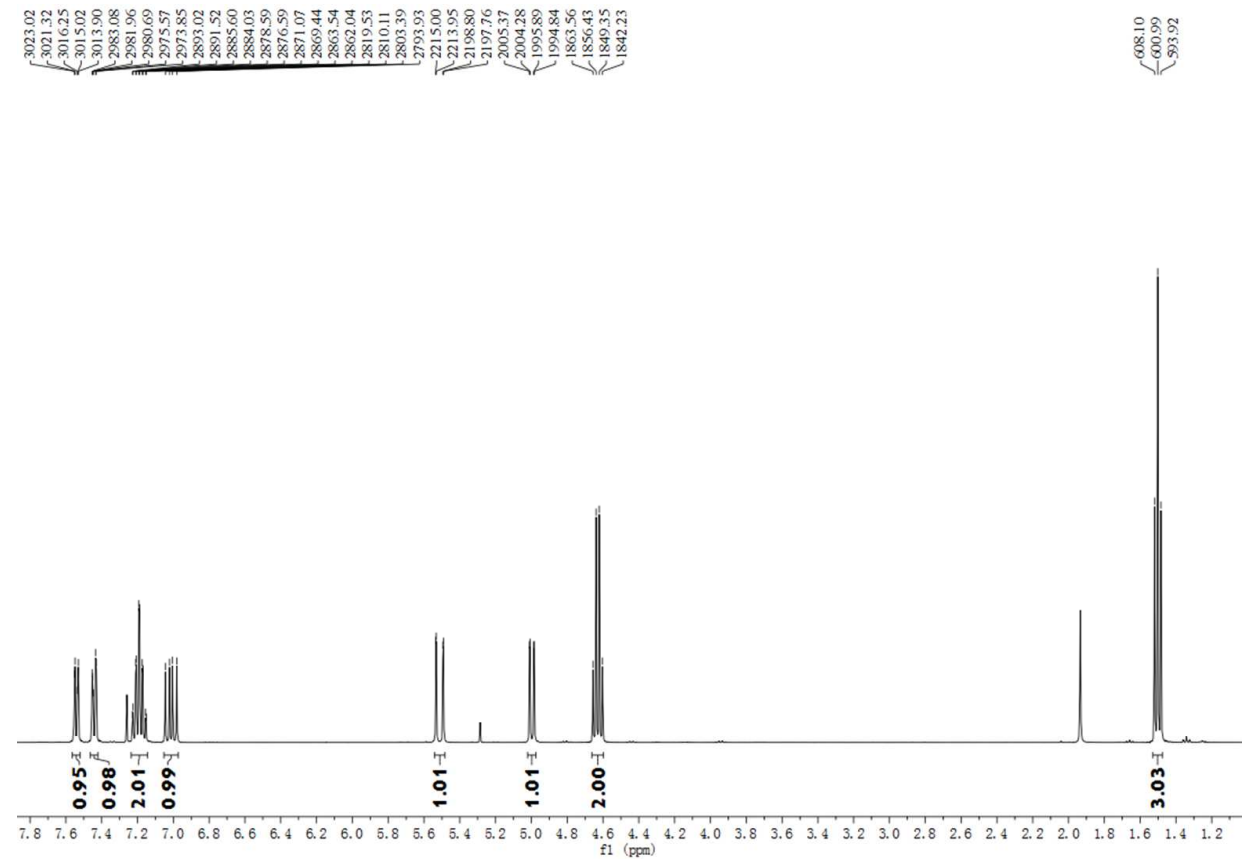

${ }^{13} \mathrm{C}$ NMR $\left(125 \mathrm{MHz}, \mathrm{CDCl}_{3}\right) \delta 156.3,140.4,132.0,127.1,122.5,121.6,117.9,110.1,101.6,66.6$, 14.6 .
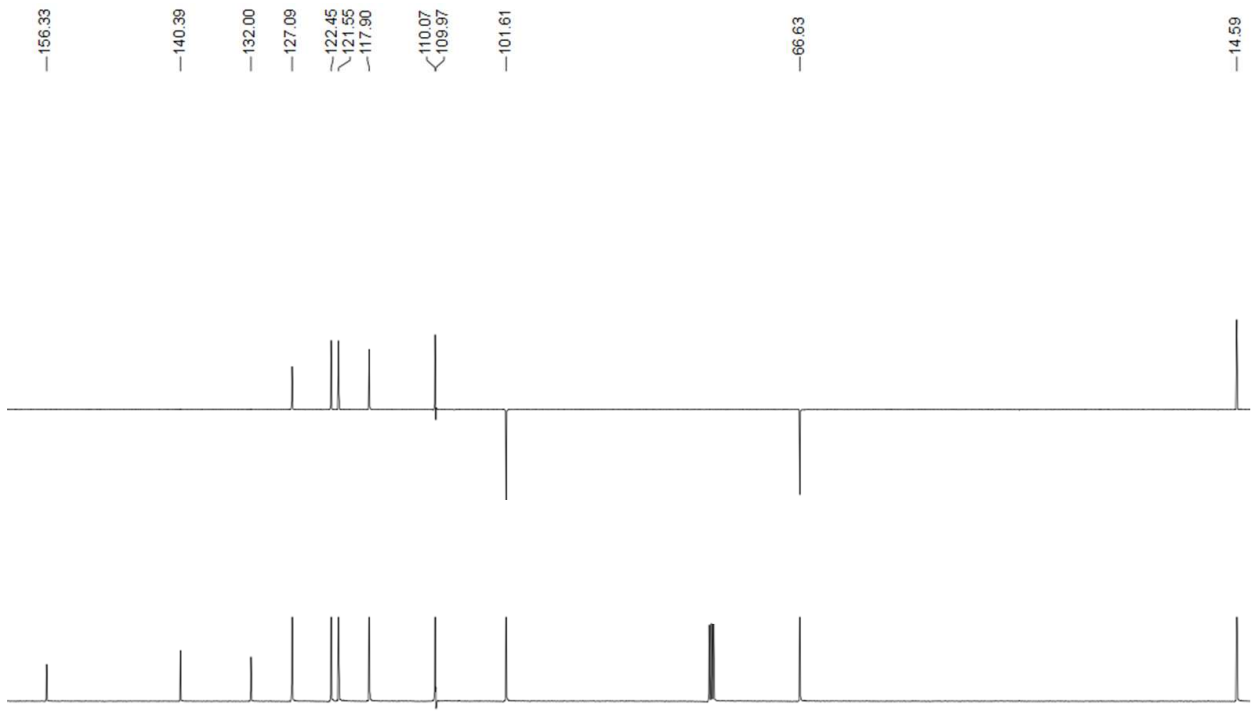

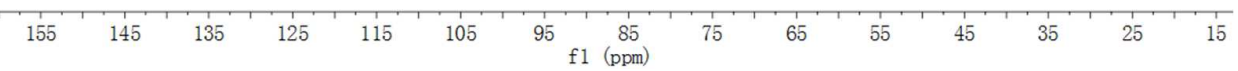


HRMS (ESI) calcd $[\mathrm{M}+\mathrm{H}]^{+}$for $\mathrm{C}_{11} \mathrm{H}_{13} \mathrm{~N}_{2} \mathrm{O} 189.1028$, found 189.1033 .

Elemental Composition Report

Page 1

Single Mass Analysis

Tolerance $=8.0$ PPM / DBE: $\min =-1.5, \max =50.0$

Element prediction: Off

Number of isotope peaks used for i-FIT $=3$

Monoisotopic Mass, Even Electron lons

90 formula(e) evaluated with 1 results within limits (up to 50 closest results for each mass)

Elements Used:

$\begin{array}{llll}\text { C: } 5-80 & \text { H: } 2-120 & \text { N: } 0-4 & \text { O: } 0-30\end{array}$

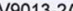

LCT PXE KE324

24-Mar-2016

16:57:47

V9013-2A_0324 28 (0.600) AM2 (Ar, 10000.0,0.00,1.00); ABS; Cm (17:29)

100

189.1033

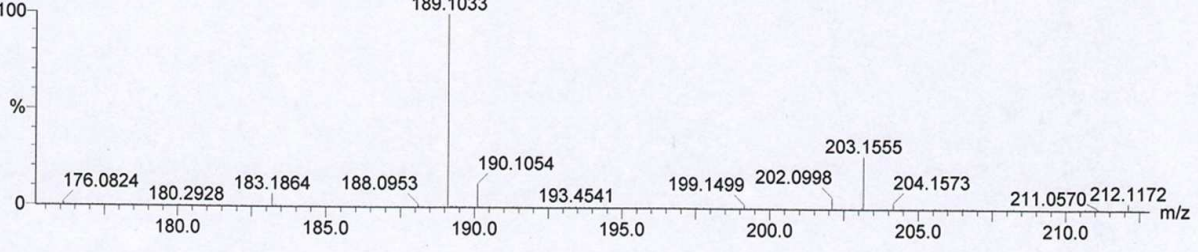

Minimum:

Maximum:

$\begin{array}{lll}5.0 & 8.0 & -1.5 \\ & & 50.0\end{array}$

Mass Calc. Mass $\mathrm{mDa}$ PPM DBE

$189.1033 \quad 189.1028$

$0.5 \quad 2.6$

6.5

i-FIT

i-FIT (Norm) Formula

$165.9 \quad 0.0$

C11 H13 N2 O 